\title{
Diagnostic performance of carbohydrate antigen $72-4$ for gastrointestinal malignancy screening in Chinese patients: a prospective study
}

\author{
Shu-Ling Wang ${ }^{1, *}$, Guan-Yu Yu ${ }^{2, *}$, An-Rong $\mathrm{Mao}^{3, *}$, Sheng-Bing Zhao ${ }^{1}$, Hui-Yun \\ Wang $^{4}$, Lun Gu${ }^{1}$, Dong Wang ${ }^{1}$, Zhao-Shen $\mathbf{L i}^{1}$ and Yu Bai ${ }^{1}$ \\ ${ }^{1}$ Department of Gastroenterology, Changhai Hospital, Second Military Medical University/Naval Medical University, Shanghai, \\ China \\ ${ }^{2}$ Department of Colorectal Surgery, Changhai Hospital, Second Military Medical University/Naval Medical University, Shanghai, \\ China \\ ${ }^{3}$ Department of Hepatic Surgery, Fudan University Shanghai Cancer Center, Department of Oncology, Shanghai Medical \\ College, Fudan University, Shanghai, China \\ ${ }^{4}$ Department of Oncology, Affiliated Hospital of Qingdao University, Qingdao, China \\ * Co-first authors \\ Correspondence to: Dong Wang, email: dr.dongwang@aliyun.com \\ Zhao-Shen Li, email: li.zhaoshen@hotmail.com \\ Yu Bai, email: baiyu1998@hotmail.com
}

Keywords: CA72-4; malignancies; gastric cancer; prediction; diagnosis

Received: July 21, $2017 \quad$ Accepted: November 03, $2017 \quad$ Published: December 05, 2017

Copyright: Wang et al. This is an open-access article distributed under the terms of the Creative Commons Attribution License 3.0 (CC BY 3.0), which permits unrestricted use, distribution, and reproduction in any medium, provided the original author and source are credited.

\section{ABSTRACT}

Background: Over the past decades, carbohydrate antigen 72-4 (CA72-4) was considered as a tumor marker that was elevated in normal people and patients with malignancies, including gastrointestinal malignancies, ovarian malignancies, endometrium malignancies, lung malignancies and so on. Besides, researches showed that elevated serum CA72-4 might predict digestive tumors, especially gastric tumors, although there was still neither sensitive nor specific tumor biomarker for gastric cancer.

Aim: This study was aimed to evaluatethe diagnostic accuracy of CA72-4 in predicting malignancies, especially the gastric cancer.

Result: The positive predictive value (PPV), negative predictive value (NPV), positive likelihood ratio (PLR) and negative likelihood ratio (NLR) of CA72-4 for diagnosing gastric cancer were $69.7 \%, 95.9 \%, 1.70$, and 0.97 , respectively. In the Receiver Operating Characteristic analysis (ROC), the area under the curvewas $\mathbf{0 . 6 2}$ for discriminating patients with gastric cancer from those without.

Conclusions: It is of little significance to perform CA72-4 testalone for predicting malignances, especially gastric cancer, in digestive disease patients.

Methods: Four hundred and three patients underwent CA72-4 test after admission to the Department of Gastroenterology in Changhai Hospital, the Second Military Medical University, from June 1, 2015 to October 31, 2015. Data of age, sex, main symptoms and final diagnosis were summarized.

\section{INTRODUCTION}

CA72-4 is a glycoprotein with high molecular weight, which is first described by Colcher in 1981 [1]. This glycoprotein was once called "tumor-associated glycoprotein-72", and could be combined with the monoclonal antibody B72-3 which came from the metastatic breasts by hybridization after utilizing an enriched extract of membranes. Since 1986, this molecule was renamed as CA72-4 and it could be detected by 
combining with the monoclonal antibody CC49 and B72-3 [2]. Up to now, it has been widely used to diagnose a variety of malignancies and to assess their progression and recurrence such as gastric cancer (GC), ovarian cancers, lung cancers and so on [3, 4]. Over the past decades, CA72-4 was emerging as a tumor marker that elevated in patients with malignancies, including gastrointestinal malignancies, ovarian malignancies, endometrium malignancies, lung malignancies, etc. $[5,6]$ and might predict digestive tumors especially gastric tumors, although it was neither sensitive nor specific tumor biomarker for GC [7-9]. As a result, doctors detect the serum level of CA72-4 in excess during daily clinical practice and the possible reasons are as followed: since the incidence of GC in East Asia is the highest especially in China $[10,11]$, Chinese people pay much attention to the early diagnosis of this malignant tumor. Endoscopic examinations and various imaging methods are reported to play a crucial role in diagnosing malignance even at early stages, thus prolonging the survival of patients suffering from GC [12]. However, due to the invasive nature of the upper endoscopy and high expenditure of imaging examination, Chinese patients prefer to undergo blood examinations for GC screening. During the past decades, with the emerging growth of economy in China, increasing Chinese people gradually attach importance to self-care. In this case, a great number of people would like to undergo CA72-4 examination during their routine health checkup. Laboratory test, routine imaging examinations and even advanced diagnostic technologies such as gastroscopy, colonoscopy, tissue biopsy as well as positron-emission tomography-computed tomography (PET-CT) are usually used to exclude various tumors once the level of serum CA72-4 is elevated. Nevertheless, most of these results are negative, contributing to the great increase in medical burden and healthcare cost. Additionally, increased CA72-4 concentration in serum is closely related to patients' excessive anxiety, which has a negative impact on health-related quality of life.

Although there were many studies about CA72-4, most of them focused on improving the accuracy of diagnosis in malignances by combination of other tumor markers [13-15], none of which showed satisfying results. Besides, most previous studies concerned about the diagnostic role of CA72-4 in digestive malignances especially in GC [16]. However, the role of CA72-4 in diagnosing GC in Chinese people was seldom investigated in recent years.

Our group has studied some biomarkers in predicting and diagnosing malignant tumors [17], and we are interested in further investigating the diagnostic value of other tumor biomarker to reduce medical cost and help clinical doctors to determine whether it is necessary to perform these tests. As a result, the main aim of our current study is to assess the diagnostic value of CA72-4 in distinguishing digestive malignances from benign disease and the role in predicting pancreatic cancer, esophagus carcinoma and other common digestive tumors in Chinese patients.

\section{RESULTS}

From June 1, 2015 to October 31, 2015, 525 patients were screened. A total of 472 patients met the inclusion criterion and 430 patients were included. Among them, three patients refused the CA72-4 examination and 427 patients received diagnostic examinations. However, 24 patients were further excluded during this period, among which fifteen patients were diagnosed with borderline tumors including seven patients with gastrointestinal stromal tumors (GISTs) and eight patients with intraductal papillary mucinous neoplasm (IPMN). As the definite diagnosis of these borderline tumors could only be confirmed by pathology, while these patients refused to undergo surgery due to the old age, therefore we could not confirm whether these tumors were benign or malignant. Since our work aimed at figuring out the role of CA72-4 in differentiating GC and other digestive malignant disease form benign diseases, we excluded the borderline tumors. In addition, six patients transferred from the Department of Medicine to the Department of Surgery, and the last three patients did not receive a certain diagnosis and was required to be followed up regularly. Finally, a total of 403 eligible patients were included in the current study, including 244 males and 159 females (Figure 1).

The basic characteristics of these subjects were described in Table 1. The average age of the patients was 55.9 years old, and subjects over 50 years old occupied $65.8 \%$. Out of 403 patients, $55.3 \%$ complained of abdominal pain, $21.1 \%$ presented with abdominal distention, and $8.2 \%$ of subjects had jaundice. Other symptoms included dysphagia, diarrhea, melena, eructation, nausea, fever and hemafecia.

\section{Final diagnosis and the level of CA72-4 in serum}

Finally, $64.0 \%$ of individuals suffered from benign disorders (Table 2). The remaining $36.0 \%$ of individuals were diagnosed with malignant tumors. Among the benign disorders, pancreatitis, polyps and cholelithiasis accounted for $31.4 \%, 27.9 \%$ and $25.2 \%$ respectively, while the rest accounted for approximately $15.5 \%$, covering esophageal leiomyoma, peptic ulcer, functional bowel disorders, chronic liver diseases, achalasia, P-J syndrome, and digestive tract obstruction. The malignance included the pancreatic cancer, liver cancer, GC, carcinoma of esophagus, cholangiocarcinoma, and colorectal cancer. Among these patients, gastric carcinoma and pancreatic cancer were the most common malignance accounting for $59.3 \%$ and $24.1 \%$ separately.

The level of CA72-4 in serum among different patients were shown in Table 2. The median level of 
CA72-4 in serum and interquartile range in 403 subjects was $1.43 \mathrm{U} / \mathrm{ml}(1.02 \mathrm{U} / \mathrm{ml}-2.82 \mathrm{U} / \mathrm{ml})$, in patients with benign diseases was $1.33 \mathrm{U} / \mathrm{ml}(0.95 \mathrm{U} / \mathrm{ml}-2.10 \mathrm{U} / \mathrm{ml})$ and in patients with malignant tumors was $1.98 \mathrm{U} / \mathrm{ml}$ (1.15 U/ml-4.58 U/ml). The median CA72-4 level in benign disorders and malignant tumors was within the normal range and there was no significant statistical difference between them $(P=0.059)$.

\section{Diagnostic accuracy}

Diagnostic accuracy of different biomarkers in GC were presented in Table 3. The sensitivity, specificity, PPV, NPV, PLR, and NLR of CA72-4 in GC were $6.97 \%, 95.90 \%, 31.58 \%, 79.17 \%, 1.70 \%$ and $0.97 \%$. The sensitivity and specificity of CA72-4 were not higher than other biomarkers in $\mathrm{GC}$, but the positive predictive value and area under curve were highest among the biomarkers. CA125 had the highest sensitivity and NPV, while AFP showed the highest specificity and positive likelihood ratio. We also described the diagnostic accuracy and its 95\% confidence interval (CI) of CA72-4 in different malignant tumors (Table 4). The sensitivity, specificity, PPV, NPV, PLR, and NLR of CA72-4 in cholangiocarcinoma were $20.00 \%, 95.48 \%, 5.26 \%, 98.96 \%, 4.42 \%, 0.84 \%$, and in liver cancer were $16.67 \%, 95.47 \%, 5,26 \%, 98.70 \%$, $3.68 \%, 0.87 \%$ respectively. However, the sensitivity of CA72-4 in colon carcinoma and carcinoma of esophagus were zero.

In the Multiple linear regression analysis, no factor was related to the concentration of CA72-4 in serum. We performed the ROC curve analysis and relevant results were shown in Figure 2: the area under the curves of the CA72-4, AFP, CA125, CEA and CA19-9 in GC

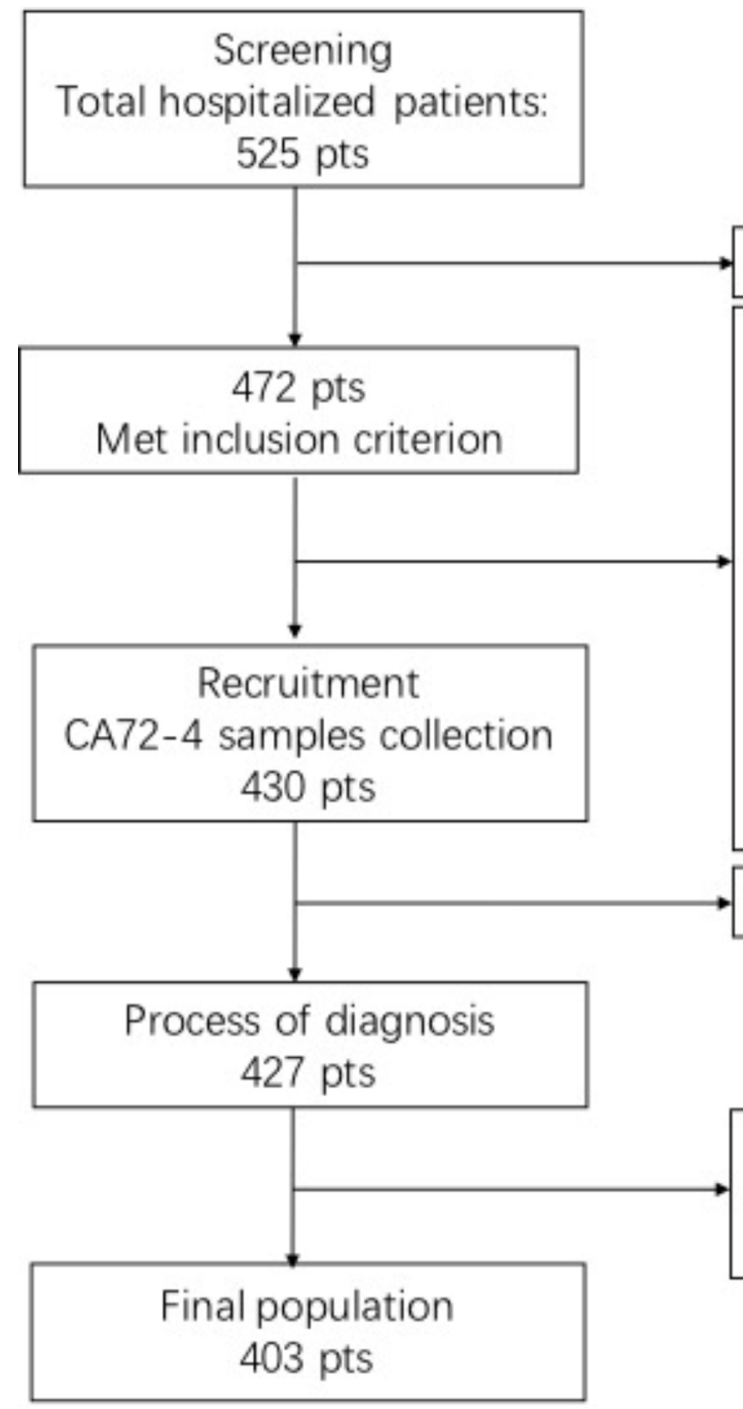

53 pts were excluded according to study aim

Reason for exclusion:

11 pts $<18$ year-old

7 pts $>85$ year-old

21 pts with Acute or severe conditions: 16 pts severe acute pancreatitis

2 pts acute suppurative cholangitis

1 pts acute heart failure

2 pts acute repiratory failure

3 pts being admitted to hospital several times during 5 months

3 pts refused to get a blood sample drawn

15 pts with borderline tumors

6 pts transferd to receive surgical treatment

3 pts with uncertain diagnosis

Figure 1: Flow chart of diagnostic test and reasons for exclusions. 
Table 1: Population characteristics $(N=403)$

\begin{tabular}{lc}
\hline Variables & No. $(\mathbf{\%})$ \\
\hline Sex & $403(100.0)$ \\
Male & $244(60.5)$ \\
Female & $159(39.5)$ \\
Age (mean \pm SD) & $55.90 \pm 14.11(18-84)$ \\
$<30$ & $22(5.3)$ \\
$30-50$ & $106(25.4)$ \\
$51-71$ & $220(52.6)$ \\
$>70$ & $55(13.2)$ \\
Main symptoms & $403(100.0)$ \\
Abdominal pain & $215(55.3)$ \\
Abdominal distention & $85(21.1)$ \\
Jaundice & $33(8.2)$ \\
Dysphagia & $17(4.2)$ \\
Diarrhea & $15(3.7)$ \\
Melena & $14(3.5)$ \\
Eructation & $12(3.0)$ \\
Nausea & $11(2.7)$ \\
Fever & $11(2.7)$ \\
Hemafecia & $8(2.0)$ \\
\hline
\end{tabular}

No. number.

patients was $0.62,0.60,0.58,0.57,0.50$, respectively. On the other hand, the area under the curves of CA724 in cholangiocarcinoma, liver cancer, gastric carcinoma, pancreatic cancer, carcinoma of the esophagus and colon carcinoma was $0.72,0.67,0.62,0.57,0.55,0.53$ respectively in Figure 3.

\section{DISCUSSION}

The major finding of our current research was that CA72-4 showed low accuracy in predicting GC for Chinese people. The sensitivity, specificity, PPV and PLR of CA72-4 examination among GC patients were not sufficient for screening.

The strengths of our study were as follows: Firstly, patients in our research were Chinese population that were poorly studied in recent years and we had some experiences in analyzing the diagnostic value of some tumor biomarkers [17]. Secondly, most previous CA724-related studies paid more attention to increasing the accuracy of GC diagnosis by combining with other tumor markers $[13,14,15]$ and there was few study concentrating on the sole diagnostic value of CA72-4 in differentiating malignant diseases. As a result, the data of our research could help clinical doctors to determine whether it is necessary to detect CA72-4, thus saving healthcare costs and reduce anxiety. Thirdly, our study was a prospective design which made the conclusion more stable and reliable. Finally, the research was carried out in a tertiary medical center where we could use various examinations to make a definite diagnose for most patients. However, this was a single-center study carried out in the Department of Gastroenterology and the sample size was relatively small, which might limit the generalization of this conclusion. Apart from this, all subjects in this research suffered from digestive diseases. To solve this problem, we also added the data of healthy control group in the Supplementary Table 1 and Supplementary Figure 1 in order to make our conclusion more reliable. These healthy participates were those who underwent routine checkups in our center. As is shown in the Supplementary Table 1, the difference of CA72-4 between healthy people and patients with malignant tumors was not statically significant (median [interval 
Table 2: Final diagnosis, median serum and interquartile range of CA72-4 level in study population

\begin{tabular}{|c|c|c|}
\hline Final diagnosis & No. $(\%)$ & CA72-4 level (U/ml) \\
\hline Benign diseases $(N=258)$ & 64.0 & $1.33(0.95-2.10)$ \\
\hline Pancreatitis & $81(31.4)$ & $1.30(0.92-1.83)$ \\
\hline Polyps (gastric, colorectal) & $72(27.9)$ & $1.36(1.02-2,22)$ \\
\hline Cholelithiasis & $65(25.2)$ & $1.34(0.96-3.09)$ \\
\hline Esophageal leiomyoma & $8(3.1)$ & $1.05(0.80-1.30)$ \\
\hline Peptic ulcer & $8(3.1)$ & $1.16(0.90-3.42)$ \\
\hline Functional bowel disorders & $6(2.3)$ & $1.17(0.92-2.84)$ \\
\hline Chronic liver diseases & $6(2.3)$ & $1.39(1.12-1.64)$ \\
\hline Achalasia & $5(1.9)$ & $1.39(1.25-1.51)$ \\
\hline P-J syndrome & $4(1.5)$ & $1.39(1.28-2.11)$ \\
\hline Digestive tract obstruction & $3(1.2)$ & $2.93(1.96-17.02)$ \\
\hline Malignant tumors $(N=145)$ & 36.0 & $1.98(1.15-4.58)$ \\
\hline Gastric carcinoma & $86(59.3)$ & $1.96(1.15-4.59)$ \\
\hline Pancreatic cancer & $35(24.1)$ & $1.88(1.10-3.19)$ \\
\hline Carcinoma of the esophagus & $8(5.5)$ & $1.79(1.20-3.68)$ \\
\hline Liver cancer & $6(4.1)$ & $3.01(1.89-6.72)$ \\
\hline Cholangiocarcinoma & $5(3.4)$ & $5.72(2.53-7.71)$ \\
\hline Colon carcinoma & $5(3.4)$ & $1.45(1.17-2.10)$ \\
\hline
\end{tabular}

CA72-4 carbohydrate antigen 72-4.

Table 3: Diagnostic accuracy and 95\% CI of biomarkers in gastric cancer

\begin{tabular}{lccccccc}
\hline $\begin{array}{l}\text { Gastric } \\
\text { cancer }\end{array}$ & Sen $(\%)$ & Spe $(\%)$ & PPV $(\%)$ & NPV $(\%)$ & PLR & NLR & AUC \\
\hline CA7-24 & 6.97 & 95.90 & 31.58 & 79.17 & 1.70 & 0.97 \\
& $(3.24,14.40)$ & $(93.11,97.59)$ & $(15.36,53.99)$ & $(74.83,82.93)$ & $(0.02,154.10)$ & $(0.95,0.99)$ & 0.62 \\
AFP & 7.69 & 98.18 & 16.67 & 95.74 & 4.23 & 0.94 & 0.60 \\
& $(1.37,33.31)$ & $(95.82,99.22)$ & $(3.01,56.35)$ & $(92.71,97.55)$ & $(0.00$, N/A) & $(0.80,1.11)$ & 1.01 \\
CEA & 9.30 & 89.59 & 19.51 & 78.45 & 0.89 & $(0.99,1.04)$ \\
& $(4.80,17.30)$ & $(85.74,92.49)$ & $(10.23,34.01)$ & $(73.93,82.38)$ & $(0.08,10.34)$ & 0.57 \\
CA19-9 & 19.77 & 79.18 & 20.48 & 78.44 & 0.95 & 1.01 \\
CA125 & $(12.72,29.40)$ & $(74.37,83.29)$ & $(13.20,30.38)$ & $(73.61,82.59)$ & $(0.58,1.56)$ & $(0.98,1.05)$ & 0.50 \\
& $(9.75,56.57)$ & $(79.06,88.18)$ & $(2.46,19.01)$ & $(92.83,98.10)$ & $(0.29,10.33)$ & $(0.68,1.11)$ & 0.58 \\
\hline
\end{tabular}

CI confidence interval, Sen sensitivity, Spe specificity, PPV positive predictive value, NPV negative predictive value, PLR positive likelihood ratio, NLR negative likelihood ratio, AUC area under curve, CA72-4 carbohydrate antigen 72-4, CA19-9 carbohydrate antigen 19-9, CA125 carbohydrate antigen 125, CEA carcino-embryonic antigen, AFP alpha fetoprotein.

ranges]: $1.14 \mathrm{U} / \mathrm{ml}[1.00 \mathrm{U} / \mathrm{ml}-1.69 \mathrm{U} / \mathrm{ml}]$ vs. $1.98 \mathrm{U} /$ $\mathrm{ml}[1.14 \mathrm{U} / \mathrm{ml}-4.58 \mathrm{U} / \mathrm{ml}], P=0.108)$. In addition, the AUC was 0.627 which meant low diagnostic value. However, the number of patients in healthy control was just 53 which might bring some bias (shown in Supplementary Figure 1 and Supplementary Table 1). In addition, patients suffering from biliary and pancreatic diseases accounted for a large proportion and there were several reasons: firstly, our department is particularly specialized in biliary and pancreatic disorders, which might attract a great proportion of patients. Secondly, the incidence of gastrointestinal tumors was not as high as reported, which was supported by our previous researches [18, 19]. Besides, the number of patients 
Table 4: Diagnostic accuracy and 95\% CI of CA72-4 in different malignant diseases

\begin{tabular}{|c|c|c|c|c|c|c|c|}
\hline CA72-4 & Sen $(\%)$ & Spe (\%) & PPV (\%) & NPV (\%) & PLR & NLR & AUC \\
\hline Gastric carcinoma & $\begin{array}{c}6.97 \\
(3.24,14.40)\end{array}$ & $\begin{array}{c}95.90 \\
(93.11,97.59)\end{array}$ & $\begin{array}{c}31.58 \\
(15.36,53.99)\end{array}$ & $\begin{array}{c}79.17 \\
(74.83,82.93)\end{array}$ & $\begin{array}{c}1.70 \\
(0.02,154.10)\end{array}$ & $\begin{array}{c}0.97 \\
(0.95,0.99)\end{array}$ & 0.62 \\
\hline Cholangiocarcinoma & $\begin{array}{c}20.00 \\
(3.62,62.45)\end{array}$ & $\begin{array}{c}95.48 \\
(92.96,97.12)\end{array}$ & $\begin{array}{c}5.26 \\
(0.94,24.64)\end{array}$ & $\begin{array}{c}98.96 \\
(97.35,99.59)\end{array}$ & $\begin{array}{c}4.42 \\
(0.00,12530.00)\end{array}$ & $\begin{array}{c}0.84 \\
(0.51,1.37)\end{array}$ & 0.72 \\
\hline Pancreatic cancer & $\begin{array}{c}2.86 \\
(0.51,14.53)\end{array}$ & $\begin{array}{c}95.11 \\
(92.40,96.88)\end{array}$ & $\begin{array}{c}5.26 \\
(0.94,24.64)\end{array}$ & $\begin{array}{c}91.15 \\
(87.88,93,59)\end{array}$ & $\begin{array}{c}0.58 \\
(0, \mathrm{~N} / \mathrm{A})\end{array}$ & $\begin{array}{c}1.02 \\
(0.96,1.08)\end{array}$ & 0.57 \\
\hline Liver cancer & $\begin{array}{c}16.67 \\
(3.01,56.35)\end{array}$ & $\begin{array}{c}95.47 \\
(92.95,97.11)\end{array}$ & $\begin{array}{c}5.26 \\
(0.94,24.64)\end{array}$ & $\begin{array}{c}98.70 \\
(96.99,99.44)\end{array}$ & $\begin{array}{c}3.68 \\
(0.00,73920.00)\end{array}$ & $\begin{array}{c}0.87 \\
(0.59,1.29)\end{array}$ & 0.67 \\
\hline Colon carcinoma & $\begin{array}{c}0.00 \\
(0.00,43.45)\end{array}$ & $\begin{array}{c}95.23 \\
(92.66,96.92)\end{array}$ & $\begin{array}{c}0.00 \\
(0.00,16.82)\end{array}$ & $\begin{array}{c}98.7 \\
(96.99,99.44)\end{array}$ & 0.00 & 1.05 & 0.53 \\
\hline $\begin{array}{l}\text { Carcinoma of the } \\
\text { esophagus }\end{array}$ & $\begin{array}{c}0.00 \\
(0.00,32.44)\end{array}$ & $\begin{array}{c}95.19 \\
(92.61,96.90)\end{array}$ & $\begin{array}{c}0.00 \\
(0.00,16.82)\end{array}$ & $\begin{array}{c}97.92 \\
(95.94,98.94)\end{array}$ & 0.00 & 1.05 & 0.55 \\
\hline
\end{tabular}

CI confidence interval, Sen sensitivity, Spe specificity, PPV positive predictive value, NPV negative predictive value, PLR positive likelihood ratio, NLR negative likelihood ratio, AUC area under curve, CA72-4 carbohydrate antigen 72-4.

suffering from liver cancer, cholangiocarcinoma and colon carcinoma was small. What's more, we did not investigate the role of CA72-4 in diagnosing other diseases involving cardiovascular, respiratory, urine or diseases from other systems.
The prognosis of early GC is better with a $90 \%$ 5 -year survival rate, while the advanced GC is relatively poor [20]. Various endoscopic examinations and radiology examinations play crucial roles in diagnosing and treating many malignance, even at early stages, which might help

roc curve

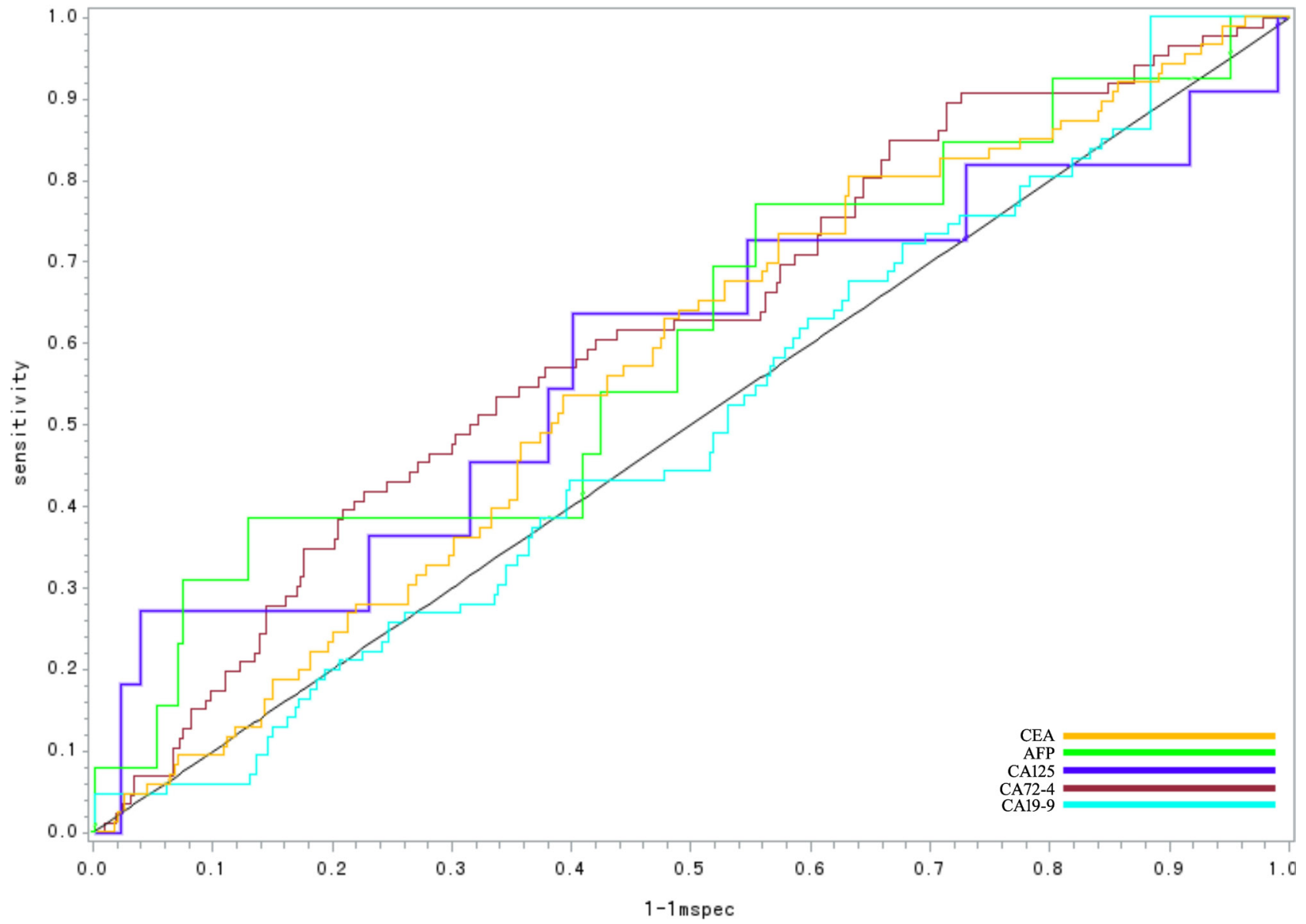

Figure 2: ROC curves and AUC of the diagnosis of tumor biomarkers for the gastric cancer. 
prolong their survival time [12]. Due to the suffering experience of the gastroscopy and high expenditure of imaging examination, Chinese patients prefer to undergo blood examinations as a way of GC screening. However, no tumor biomarker was proposed to screen, diagnose or monitor the prognosis of GC in the European Society for Medical Oncology Clinical Recommendations and the National Comprehensive Cancer Network Clinical Practice for GC 2013 [21]. However, Chen et al. reported that CA724 was the most relevant serum tumor biomarker in serum for GC in Chinese population [22]. Since it was a systematic review and meta-analysis study rather than a prospective study with high quality, the result might be affected by the result of individual study. Actually, the heterogeneity was much high which was indicated by the $I^{2}(77 \%)$, resulting a less reliable conclusion in that study. In addition, it involved many multiple single test, which meant different measurements and cutoff were used to assay the level of CA72-4 in serum. As a result, this article had weak recommendation strength in spite of larger sample size. Our group has studied the role of CA125 in diagnosing gastrointestinal malignancy in male patient and its role in predicting serous effusion [17] and we want to further study whether CA72-4 can play its role in predicting and diagnosing malignancies.

Although CA72-4 has been reported to be the most sensitive and specific biomarker for GC [16], the predictive accuracy is still low and couldn't meet the requirement of clinical practice. Yoon et al. declared that chemo-sensitivity of GC patients was not associated with pretreatment CA72-4 levels [23]. Although the elevated concentrations of CA72-4 in the peritoneal washing of GC patients were closely associated with the involvement of lymph nodes, serosa organ invasion and more advanced condition of disease, levels of CA72-4 in serum did not show a close link as mentioned above [24]. All results of researches mentioned above are in agreement with ours. Our research found that the mean CA72-4 concentration in serum of patients with benign diseases and malignant tumors showed no statistically different $(P=0.059)$, and both of them were in the normal range of CA72-4. According the data, we concluded that CA72-4 couldn't differentiate malignancy from benign. From the data in Table 3, although the positive predictive value and area

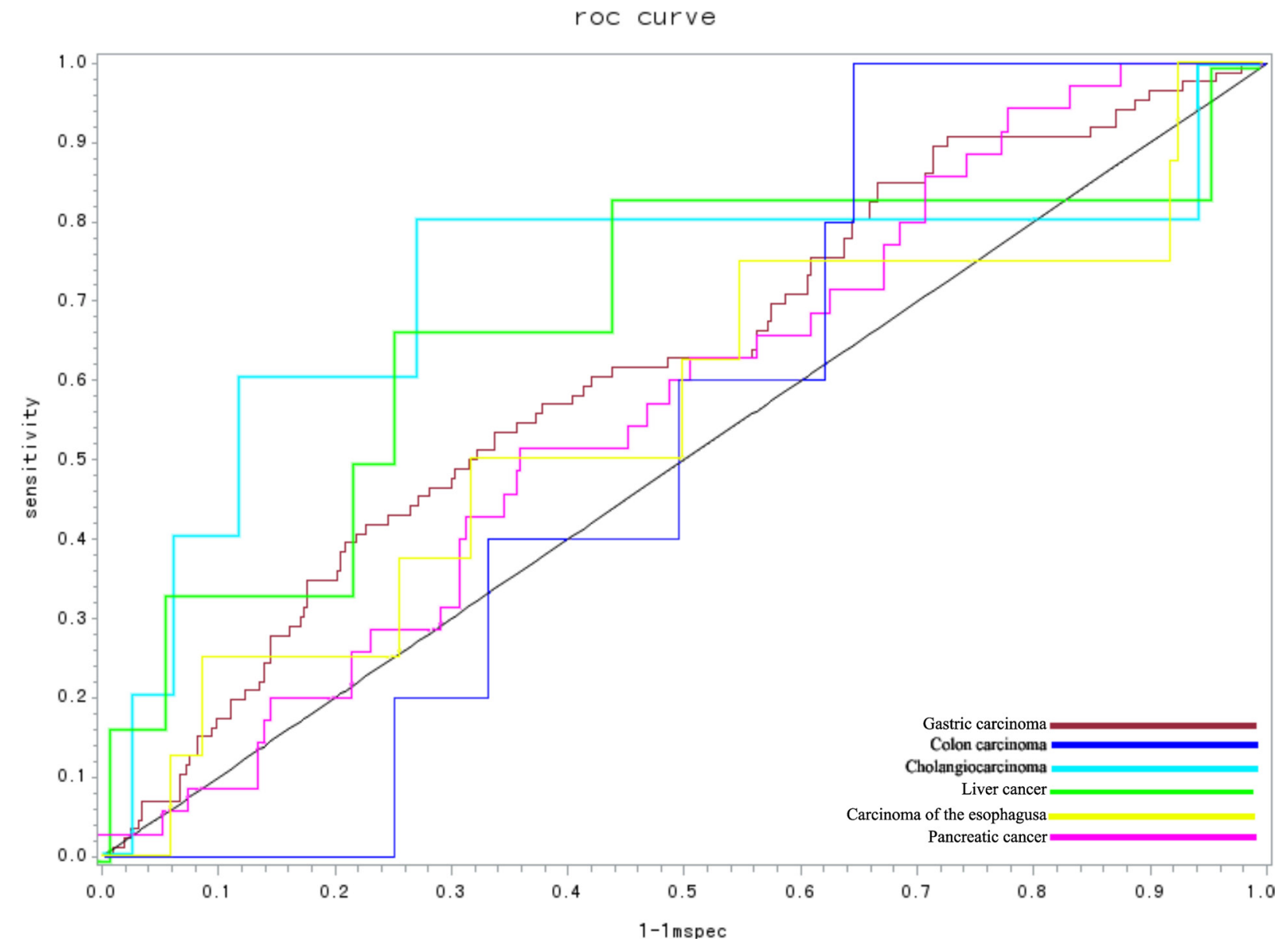

Figure 3: ROC curves and AUC of the diagnosis of CA72-4 for the malignant tumors. 
under curve were highest among the biomarkers, the sensitivity and specificity of CA72-4 were not higher than other biomarkers in GC. To further study whether CA72-4 played a role in diagnosing other common digestive cancers, we calculated the diagnostic accuracy of CA72-4 in different malignant diseases as shown in Table 4. We found that the sensitivity in diagnosing cholangiocarcinoma and liver cancer was higher than in gastric carcinoma, but this result might be caused by their small sample size. In addition, the sensitivity and specificity of colon carcinoma and carcinoma of the esophagus was non-significant. All of these findings indicate that CA72-4 alone shows less value in diagnosing malignancy especially in GC than physicians thought.

Recent studies found that CA72-4 played a significant role in pancreatic disease. Jiang et al. reported that the level of CA72-4 in serum was relatively higher in patients with pancreatic cancer compared with those suffering from pancreatic benign disease [25]. Recently, Liu et al. demonstrated that the concentration of CA72-4 was significantly correlated with the poor progression and organ invasion of pancreatic adenocarcinoma. It was also reported that smoking patients had much higher serum CA72-4 levels $(P=0.003)$. Furthermore, they found those with higher level of serum CA72-4 had 2.34 times the risk of death compared with those with normal level of serum CA72-4 [26]. As mentioned before, our department is professional in the biliary and pancreatic diseases. We involved 81 patients with pancreatitis and 35 patients with pancreatic cancer in our current study. The median serum CA72-4 level in pancreatitis and pancreatic cancer were $1.30 \mathrm{U} / \mathrm{ml}$ and $1.88 \mathrm{U} / \mathrm{ml}$, and the difference between them was also not statistically significant $(P=0.1677)$. According to our data, the diagnostic value of CA72-4 in pancreas related diseases is very low. In addition, an elevation of serum CA72-4 was reported when patients took Ganoderma Lucidum Spore Powder [27]. In our study, the medical history of all patients was collected on admission, and none of the patients received these medications. Although CA72-4 is seldom expressed in the normal adult and patients with benign tumors, some researchers focus on the relationship between CA72-4 level and benign diseases, thus resulting in the widespread of CA72-4 in daily clinical practice. Although Li et al. found that changes of serum CA72-4 concentration might help assess the sensitivity to chemotherapy [28], we didn't find significant difference in benign diseases. In addition, the Multiple linear regression analysis was also applied to investigate the factors including age, sex and final diagnosis which might be associated with the CA72-4 concentration. Unfortunately, no factor was associated with serum CA72-4 level.

\section{CONCLUSIONS}

It is of little importance to perform CA72-4 detection alone to screen gastrointestinal malignancy in patients with digestive symptoms. CA72-4 shows less value in diagnosing GC and couldn't meet the requirement of clinical practice.

\section{MATERIALS AND METHODS}

\section{Study population}

From June 1, 2015 to October 31, 2015, we prospectively screened 525 consecutive hospitalized patients in the Department of Gastroenterology of Changhai Hospital, the Second Military Medical University. The inclusion criterion was patients whose digestive symptom was a chief complaint. Finally, 53 patients complaining of non-digestive symptoms were ruled out, leaving 472 digestive symptoms-related patients. Exclusion criteria were as follows: $<18$ or $>85$-year-old, acute and severe patients (severe acute pancreatitis, severe acute suppurative cholangitis, and severe cardiovascular or pulmonary comorbidities), transferring to other department for surgical treatment, being admitted to hospital several times during June to October 2015. Figure 1 describes the study protocol and reasons for exclusions. The final population of the study were 403 patients, 244 males and 159 females. Data of patients' age, main symptoms, final diagnosis and median serum CA72-4 level were summarized in Tables 1 and 2 .

The study protocol was approved by the Shanghai Changhai Hospital Ethics Committee, and the study practice was in accordance with the Declaration of Helsinki with written informed consent obtained from all participants.

\section{Measurement of biomarkers in serum specimens}

Blood samples were collected from all the participants after an overnight fast. All the remaining patients received CA72-4, carbohydrate antigen 19-9 (CA19-9) and carcino-embryonic antigen (CEA) test, 288 patients underwent alpha fetoprotein (AFP) test and 257 patients received carbohydrate antigen 125 (CA125) test. Samples were collected before the treatment. Four milliliters of venous blood were drawn for testing. All samples were obtained by experienced nurses and assayed at the Department of Clinical Laboratory of Changhai Hospital. Concentrations of CA724 were measured by Electrochemiluminescence using the Roche combas e602 analyzer, other markers measured by chemiluminescent micro particle immunoassay (CMIA) using the Abbott ARCHITECT i2000sr chemiluminescence analyzer. The recommended upper cutoff values for CA72-4, CA19-9, CEA, CA125 and AFP were $9.8 \mathrm{U} / \mathrm{ml}, 37 \mathrm{U} / \mathrm{ml}, 5 \mathrm{ng} / \mathrm{ml}$, $35 \mathrm{U} / \mathrm{ml}$ and $8.78 \mathrm{ng} / \mathrm{ml}$, separately. 


\section{Process of diagnosis}

All the subjects underwent chest X-ray, electrocardiogram and routine abdominal ultrasound examination of the liver, gallbladder, pancreas, spleen, kidneys, ascites, and pelvic fluids after being admitted to hospital. Further examinations were performed as needed based on the initial diagnosis in order to make a final diagnosis. Patients with abdominal pain could receive some examinations such as gastroscopy, colonoscopy, computed tomography (CT), magnetic resonance imaging (MRI), and even three-dimensional imaging of the small bowel to confirm the location, pathology and to evaluate the severity of illness. For patients with jaundice, magnetic resonance cholangiopancreatography (MRCP), CT, and/or endoscopic ultrasonography (EUS), fine-needle aspiration (FNA), and endoscopic retrograde cholangiopancreatography (ERCP) were applied to find the cause of jaundice. For patients with serous effusion, diagnostic paracentesis and thoracentesis were performed.

Once malignancy was suspected, biopsy should be performed to confirm the final diagnosis. For those without biopsy or with uncertain biopsy results, the final diagnosis was made by a combination of history, physical examination, imaging tests, endoscopy tests, serologic tests, and follow-up of these patients. Borderline tumors such as intraductal papillary mucinous neoplasm (IPMN) and gastrointestinal stromal tumors (GISTs) were ruled out from the study.

All the imaging tests were performed in the Department of Radiology by experienced operators. The endoscopic procedures were carried out by endoscopists of the Digestive Endoscopy Center of Changhai Hospital. Besides, the medical history of all patients was collected on admission, to verify whether they received medications which could elevate the serum level of CA72-4.

\section{Statistical analysis}

All the data was analyzed using SAS version 9.3 for windows. The Kolmogorov-Smirnov test was used to assess the normality of total CA72-4 levels. Two independent groups were assessed by Levene's test for equality of variances. Continuous variables were expressed as mean \pm standard deviation and range, whereas CA724 values were presented as median. Categorical variables were expressed as a percentage and were analyzed with Mann-Whitney $U$ test. The diagnostic value of biomarkers for detection of GC was expressed as sensitivity, specificity, positive likelihood ratio, negative likelihood ratio, positive predictive value, negative predictive value, and the corresponding 95\% confidence interval. Multiple linear regression analysis was applied to investigate the factors associated with the CA72-4 concentration. The factors included age, sex and final diagnosis. In addition, receiver operating characteristic (ROC) analysis was used to evaluate the diagnostic value of biomarkers and area under the curve (AUC) was calculated. Twotailed probability value of $<0.05$ was considered to be statistically significant.

\section{Abbreviations}

CA72-4: carbohydrate antigen 72-4; GC: gastric cancer; PPV: positive predictive value; NPV: negative predictive value; PLR: positive likelihood ratio; NLR: negative likelihood ratio; ROC: receiver operating characteristic; AUC: analysis and the area under the curve; PET-CT: positron-emission tomography-computed tomography; CA19-9: carbohydrate antigen 19-9; CA125: carbohydrate antigen 125; CEA: carcino-embryonic antigen; AFP: alpha fetoprotein.

\section{ACKNOWLEDGMENTS}

Dr. Bai Yu is supported by 2014 Shanghai RisingStar Program (A type, No. 14QA1404800), Outstanding Young Scholars Fund of Second Military Medical University, the National Natural Science Foundation of China (Grant No. 81670473) and also supported by Three engineering training funds in Shenzhen, No. SYLY201718. For the remaining authors, none was declared. We thank all the doctors and nurses who helped to manage the patients who underwent this clinical test in our department during the months of study.

\section{CONFLICTS OF INTEREST}

No conflicts of interest exist for all authors.

\section{REFERENCES}

1. Colcher D, Hand PH, Nuti M, Schlom J. A spectrum of monoclonal antibodies reactive with human mammary tumor cells. Proc Natl Acad Sci U S A. 1981; 78:3199-203.

2. Johnson VG, Schlom J, Paterson AJ, Bennett J, Magnani JL, Colcher D. Analysis of a human tumor-associated glycoprotein (TAG-72) identified by monoclonal antibody B72.3. Cancer Res. 1986; 46:850-57.

3. Yamamoto M, Yoshinaga K, Matsuyama A, Tsutsui S, Ishida T. CEA/CA72-4 levels in peritoneal lavage fluid are predictive factors in patients with gastric carcinoma. J Cancer Res Clin Oncol. 2014; 140:607-12.

4. Lenhard MS, Nehring S, Nagel D, Mayr D, Kirschenhofer A, Hertlein L, Friese K, Stieber P, Burges A. Predictive value of CA 125 and CA 72-4 in ovarian borderline tumors. Clin Chem Lab Med. 2009; 47:537-42.

5. Udagawa Y, Aoki D, Ito K, Uejima T, Uemura M, Nozawa S. Clinical characteristics of a newly developed ovarian tumour marker, galactosyltransferase associated with tumour (GAT). Eur J Cancer. 1998; 34:489-95. 
6. Cramer DW, Bast RC Jr, Berg CD, Diamandis EP, Godwin AK, Hartge P, Lokshin AE, Lu KH, McIntosh MW, Mor G, Patriotis C, Pinsky PF, Thornquist MD, et al. Ovarian cancer biomarker performance in prostate, lung, colorectal, and ovarian cancer screening trial specimens. Cancer Prev Res (Phila). 2011; 4:365-74. https://doi.org/10.1158/1940-6207. CAPR-10-0195.

7. Li Y, Yang Y, Lu M, Shen L. Predictive value of serum CEA, CA19-9 and CA72.4 in early diagnosis of recurrence after radical resection of gastric cancer. Hepatogastroenterology. 2011; 58:2166-70. https://doi.org/10.5754/hge11753.

8. Bornschein J, Selgrad M, Wex T, Kuester D, Malfertheiner P. Serological assessment of gastric mucosal atrophy in gastric cancer. BMC Gastroenterol. 2012; 12:10. https://doi. org/10.1186/1471-230X-12-10.

9. Zur B, Holdenrieder S, Walgenbach-Brünagel G, Albers E, Stoffel-Wagner B. Method comparison for determination of the tumor markers AFP, CEA, PSA and free PSA between Immulite 2000 XPI and Dimension Vista 1500. Clin Lab. 2012; 58:97-105.

10. Fock KM. Review article: the epidemiology and prevention of gastric cancer. Aliment Pharmacol Ther. 2014; 40:250-60.

11. Ferlay J, Soerjomataram I, Dikshit R, Eser S, Mathers C, Rebelo M, Parkin DM, Forman D, Bray F. Cancer incidence and mortality worldwide: sources, methods and major patterns in GLOBOCAN 2012. 2015; 136:E359-86.

12. Pan Z, Pang L, Ding B, Yan C, Zhang H, Du L, Wang B, Song Q, Chen K, Yan F. Gastric cancer staging with dual energy spectral CT imaging. PLoS One. 2013; 8:e53651. https://doi. org/10.1371/journal.pone.0053651.

13. Yang AP, Liu J, Lei HY, Zhang QW, Zhao L, Yang GH. CA72-4 combined with CEA, CA125 and CA19-9 improves the sensitivity for the early diagnosis of gastric cancer. Clin Chim Acta. 2014; 437:183-6.

14. Sun Z, Zhang N. Clinical evaluation of CEA, CA19-9, CA72-4 and CA125 in gastric cancer patients with neoadjuvant chemotherapy. World J Surg Oncol. 2014; 12:397. https://doi. org/10.1186/1477-7819-12-397.

15. Zhu YB, Ge SH, Zhang LH, Wang XH, Xing XF, Du H, Hu Y, Li YA, Jia YN, Lin Y, Fan B, Ji JF. [Clinical value of serum CEA, CA19-9, CA72-4 and CA242 in the diagnosis and prognosis of gastric cancer]. Zhonghua Wei Chang Wai Ke Za Zhi. 2012; 15:161-4.

16. Guadagni F, Roselli M, Amato T, Cosimelli M, Perri P, Casale V, Carlini M, Santoro E, Cavaliere R, Greiner JW, et al. CA 72-4 measurement of tumor-associated glycoprotein 72 (TAG72 ) as a serum marker in the management of gastric carcinoma. Cancer Res. 1992; 52:1222-27.

17. Zhu X, Zhou W, Chen Y, Gao J, Kong F, Liu J, Li Z, Gao J, Bai Y. High serum carbohydrate antigen 125 concentration can predict serous effusion but not gastrointestinal malignancy in male patients. Tumour Biol. 2014; 35:5129-35.
18. Bai Y, Xu C, Zou DW, Gao J, Li ZS. Diagnostic accuracy of features predicting lower gastrointestinal malignancy: a colonoscopy database review of 10,603 Chinese patients. Colorectal Dis. 2011; 13:658-62.

19. Bai Y, Li ZS, Zou DW, Wu RP, Yao YZ, Jin ZD, Ye P, Li SD, Zhang WJ, Du YQ, Zhan XB, Liu F, Gao J, Xu GM. Alarm features and age for predicting upper gastrointestinal malignancy in Chinese patients with dyspepsia with high background prevalence of Helicobacter pylori infection and upper gastrointestinal malignancy: an endoscopic database review of 102,665 patients from 1996 to 2006. Gut. 2010; 59:722-28.

20. Chen XZ, Hu JK, Zhou ZG, Rui YY, Yang K, Wang L, Zhang B, Chen ZX, Chen JP. Meta-analysis of effectiveness and safety of D2 plus para-aortic lymphadenectomy for resectable gastric cancer. J Am Coll Surg. 2010; 210:100-05.

21. Ajani JA, Bentrem DJ, Besh S, D’Amico TA, Das P, Denlinger C, Fakih MG, Fuchs CS, Gerdes H, Glasgow RE, Hayman JA, Hofstetter WL, Ilson DH, et al, and National Comprehensive Cancer Network. Gastric cancer, version 2.2013: featured updates to the NCCN Guidelines. J Natl Compr Canc Netw. 2013; 11:531-46.

22. Chen XZ, Zhang WK, Yang K, Wang LL, Liu J, Wang L, Hu JK, Zhang B, Chen ZX, Chen JP, Zhou ZG, Mo XM. Correlation between serum CA724 and gastric cancer: multiple analyses based on Chinese population. Mol Biol Rep. 2012; 39:9031-9. https://doi.org/10.1007/s11033-012-1774-x.

23. Yoon SN, Roh SA, Cho DH, Kim MB, Hyun YL, Ro S, Kim BS, Kim SY, Kim YS, Kim JC. In vitro chemosensitivity of gastric adenocarcinomas to histone deacetylase inhibitors, compared to established drugs. Hepatogastroenterology. 2010; 57:657-62.

24. Fernandes LL, Martins LC, Nagashima CA, Nagae AC, Waisberg DR, Waisberg J. CA72-4 antigen levels in serum and peritoneal washing in gastric cancer. Correlation with morphological aspects of neoplasia. Arq Gastroenterol. 2007; 44:235-39.

25. Jiang XT, Tao HQ, Zou SC. Detection of serum tumor markers in the diagnosis and treatment of patients with pancreatic cancer. Hepatobiliary Pancreat Dis Int. 2004; 3:464-68.

26. Liu P, Zhu Y, Liu L. Elevated serum CA72-4 levels predict poor prognosis in pancreatic adenocarcinoma after intensitymodulated radiation therapy. Oncotarget. 2015; 6:9592-9. https://doi.org/10.18632/oncotarget.3562.

27. Liang Y, He M, Fan X, Ye W, Yang Z, Zhong R. An abnormal elevation of serum CA72-4 by ganoderma lucidum spore powder. Ann Clin Lab Sci. 2013; 43:337-40.

28. Zou L, Qian J. Decline of serum CA724 as a probable predictive factor for tumor response during chemotherapy of advanced gastric carcinoma. Chin J Cancer Res. 2014; 26:404-9. https:// doi.org/10.3978/j.issn.1000-9604.2014.07.02. 